\title{
Air Traffic Sector Configuration Change Frequency
}

\section{Gano Chatterji and Michael Drew}

\author{
University of California, Santa Cruz \\ NASA Ames Research Center \\ Moffett Field, CA 94035
}




\section{Sector Change Frequency}
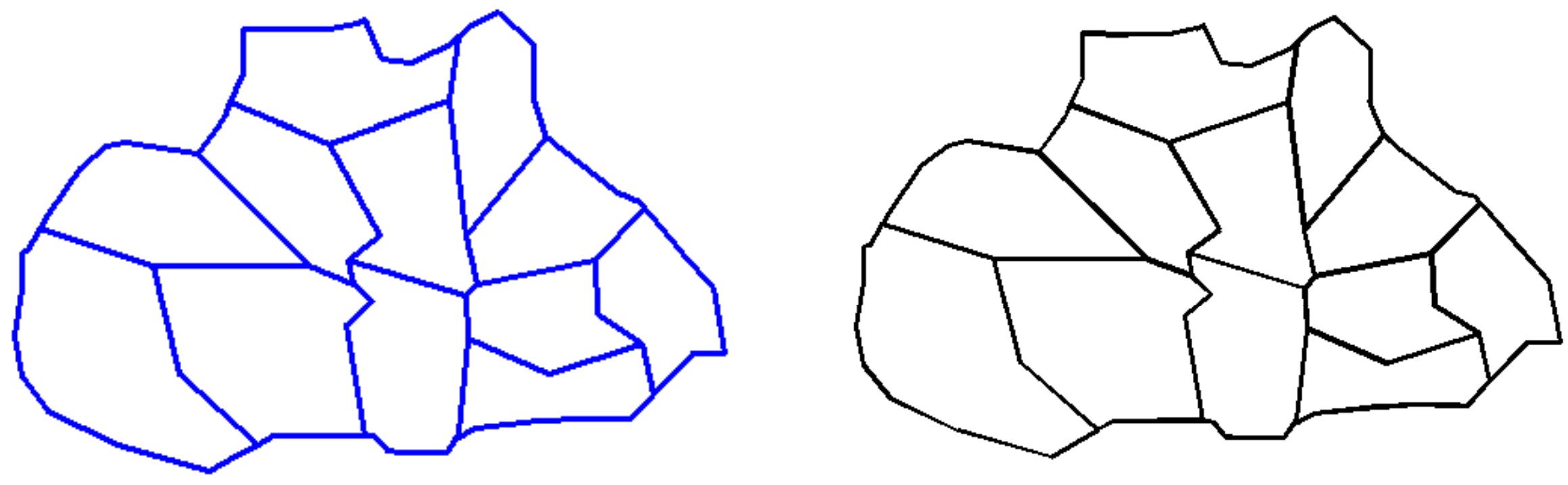


\section{Main Points}

- Sectors created with traffic from good-weather days can be used on other good-weather days.

- Sector configurations created for shorter periods (two-hours) can be used for longer periods (six to 12 hours).

- One to three sector configurations are adequate for a day. 


\section{Outline}

- Air Traffic Dataset

- Robust Sectorization Method

- Validation Results

- Conclusions

- Future Work 


\section{Air Traffic Dataset (206 Days 2007)}

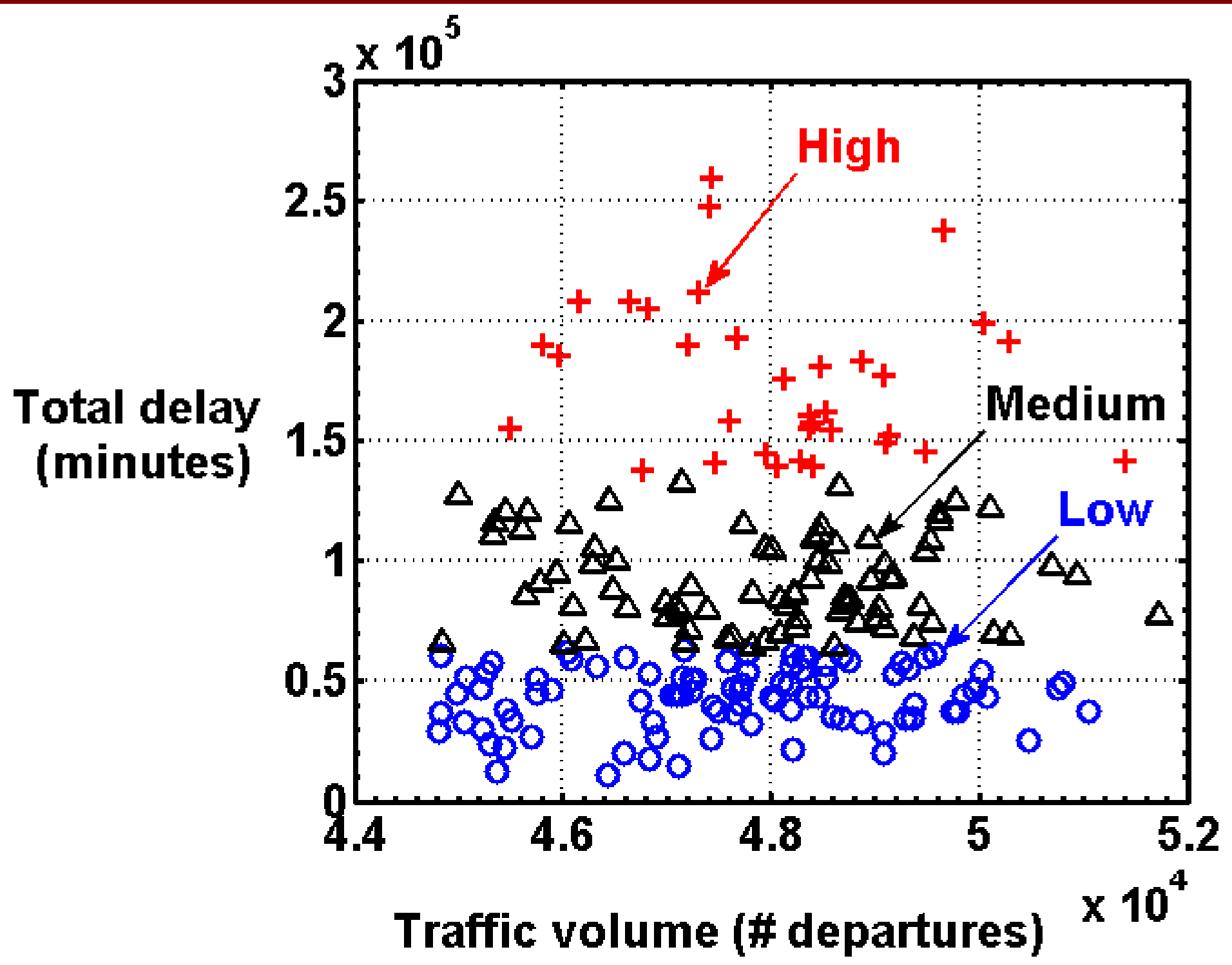




\section{Air Traffic Dataset}

20 high-volume good-weather days from 2007

- 10 days in training set

- 10 days in test set 


\section{Air Traffic Dataset}

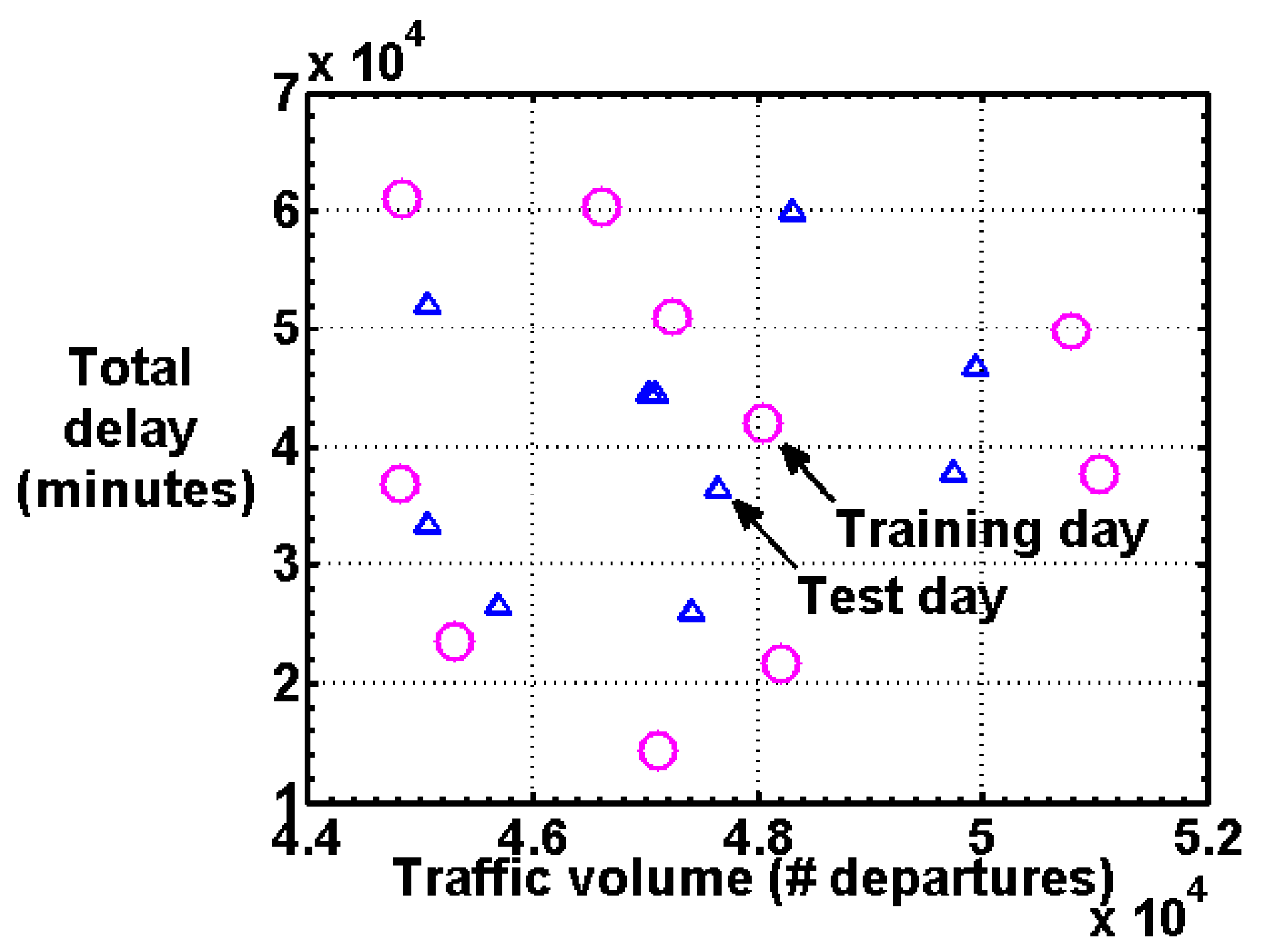




\section{Training Set Data Bounds}

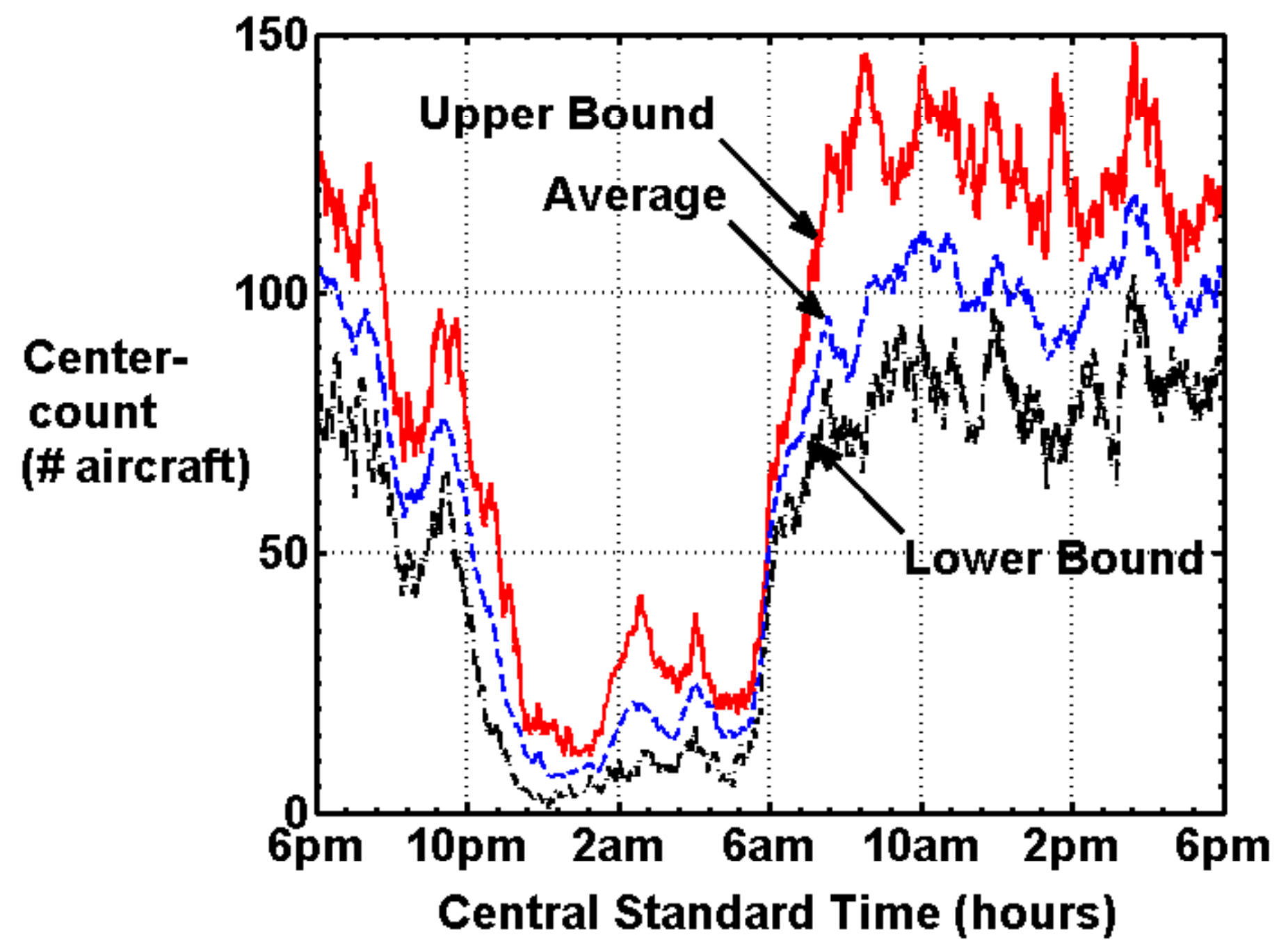




\section{Sectorization Method}
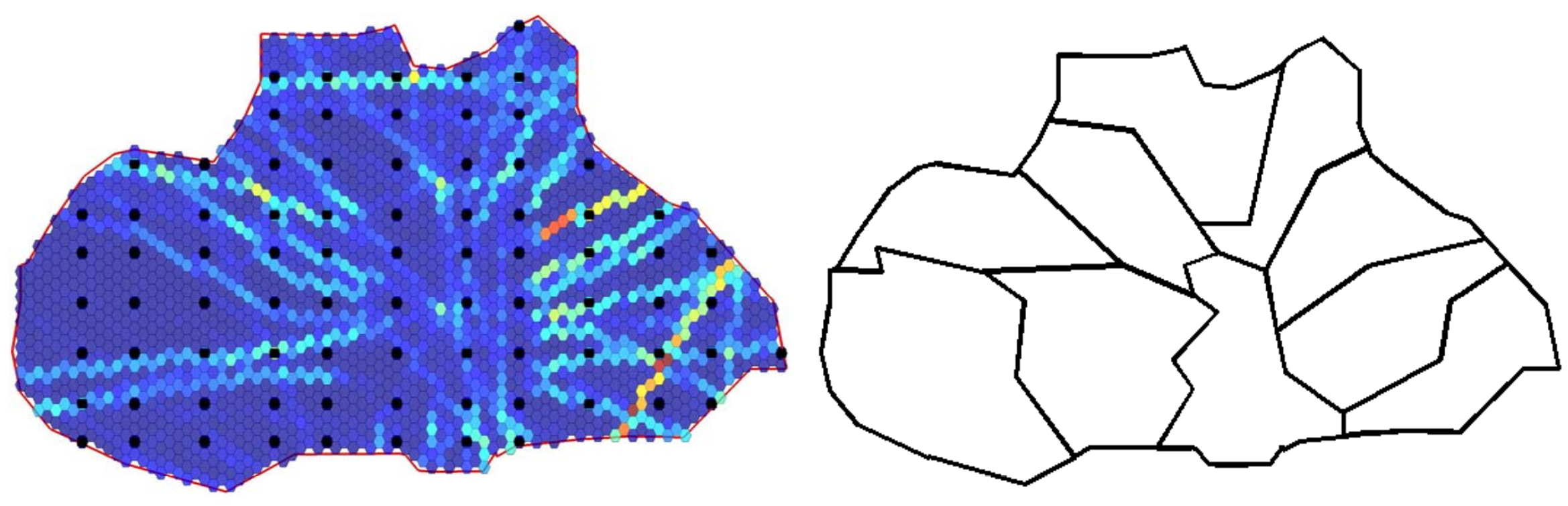

- Airspace discretized with hexagonal tiles.

- Mixed Integer Linear Programming used for grouping tiles into sectors.

- Boundary smoothing algorithm used for final sector boundaries. 


\section{Robust Sectorization Method}

1. Break 24-hours traffic data from training set into twohour periods.

2. Create nine sector configurations for each two-hour period by varying number of sectors.

3. Create nine histograms using traffic data and sector configurations for each two-hour period.

4. Select one configuration for each two-hour period based on histograms.

5. Select few sector configurations for the entire day. 


\section{Sector Configuration Selection}
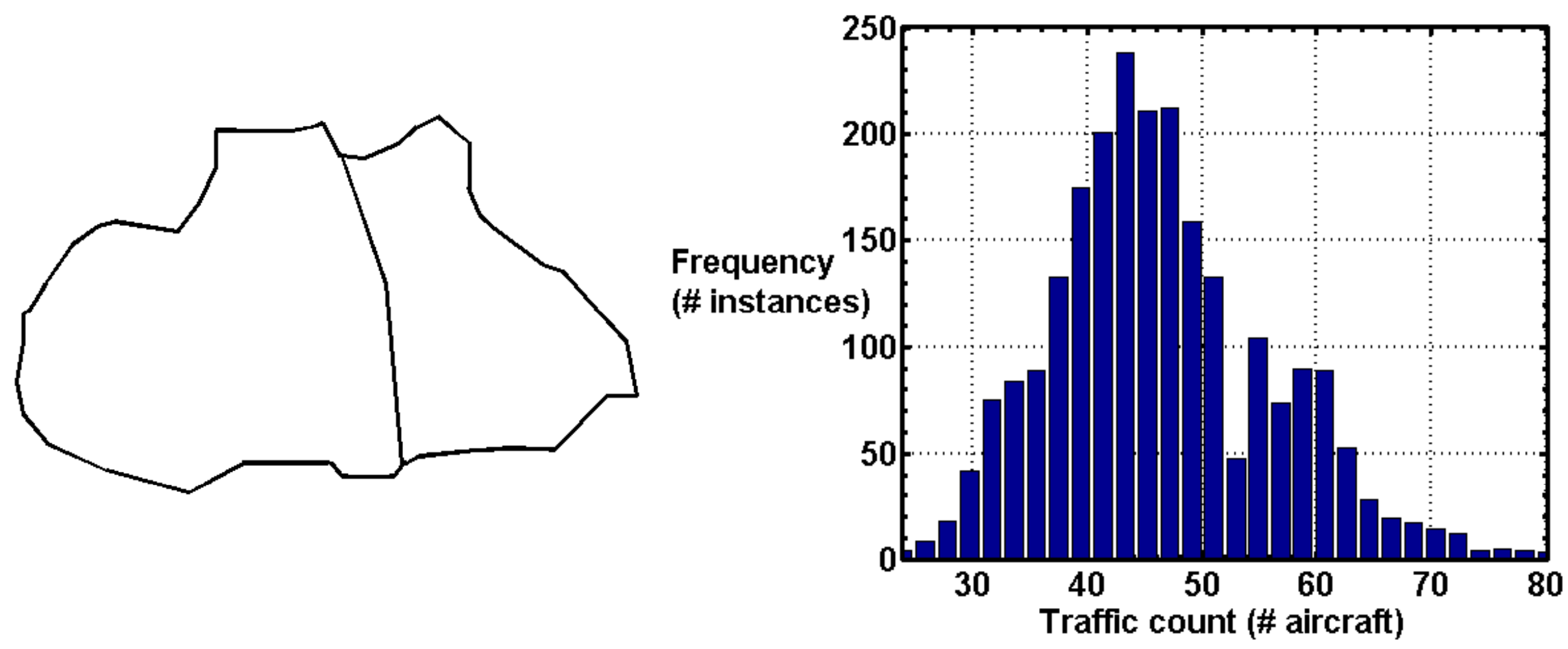

Histogram of traffic counts during 6:00 - 8:00 pm Central Standard Time with airspace partitioned into two sectors based on 10 training days of traffic. 


\section{Sector Configuration Selection}
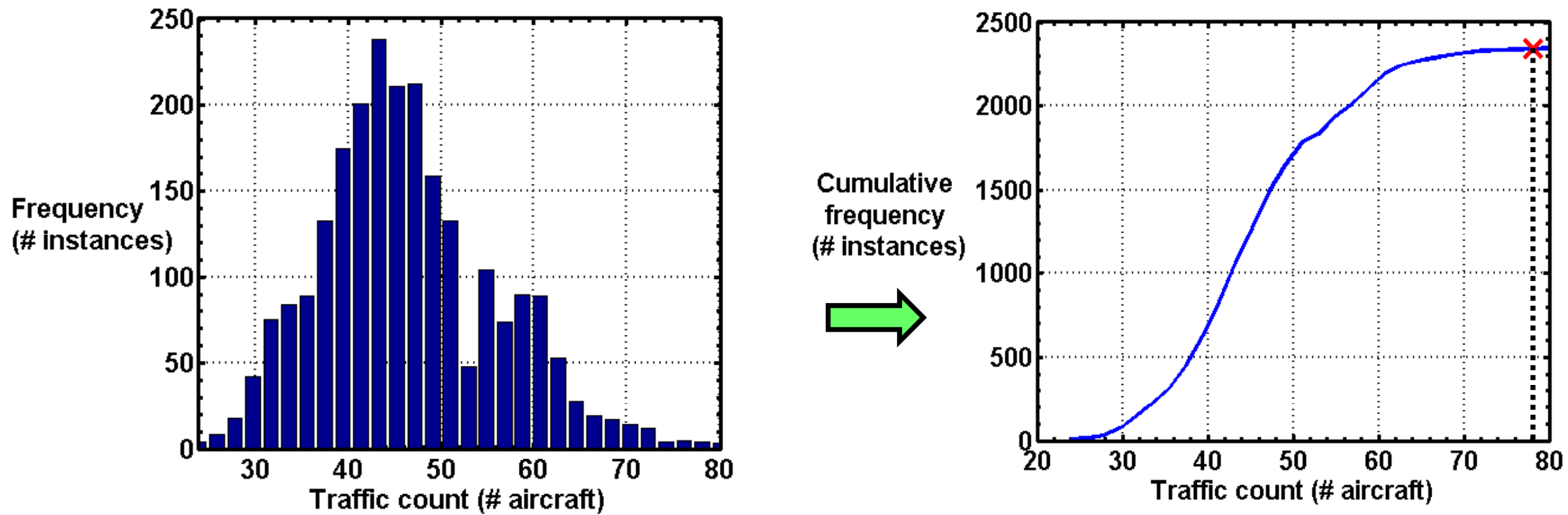

- 99.9 percentile value is 2344 .

- Traffic count corresponding to 2344 is 78 aircraft. 


\section{Sector Configuration (18 Sectors)}
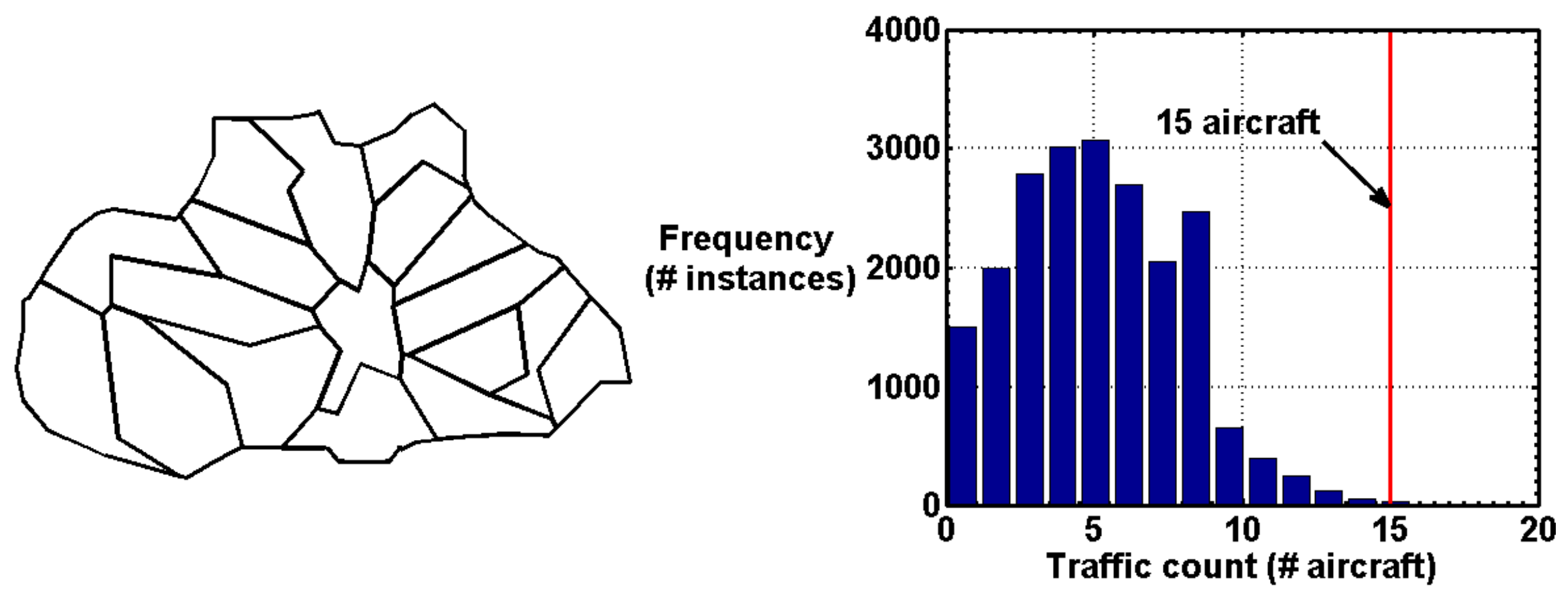

Histogram of traffic counts during 6:00 - 8:00 pm Central Standard Time with airspace partitioned into 18 sectors based on 10 training days of traffic. 


\section{Sector Configuration Selection}

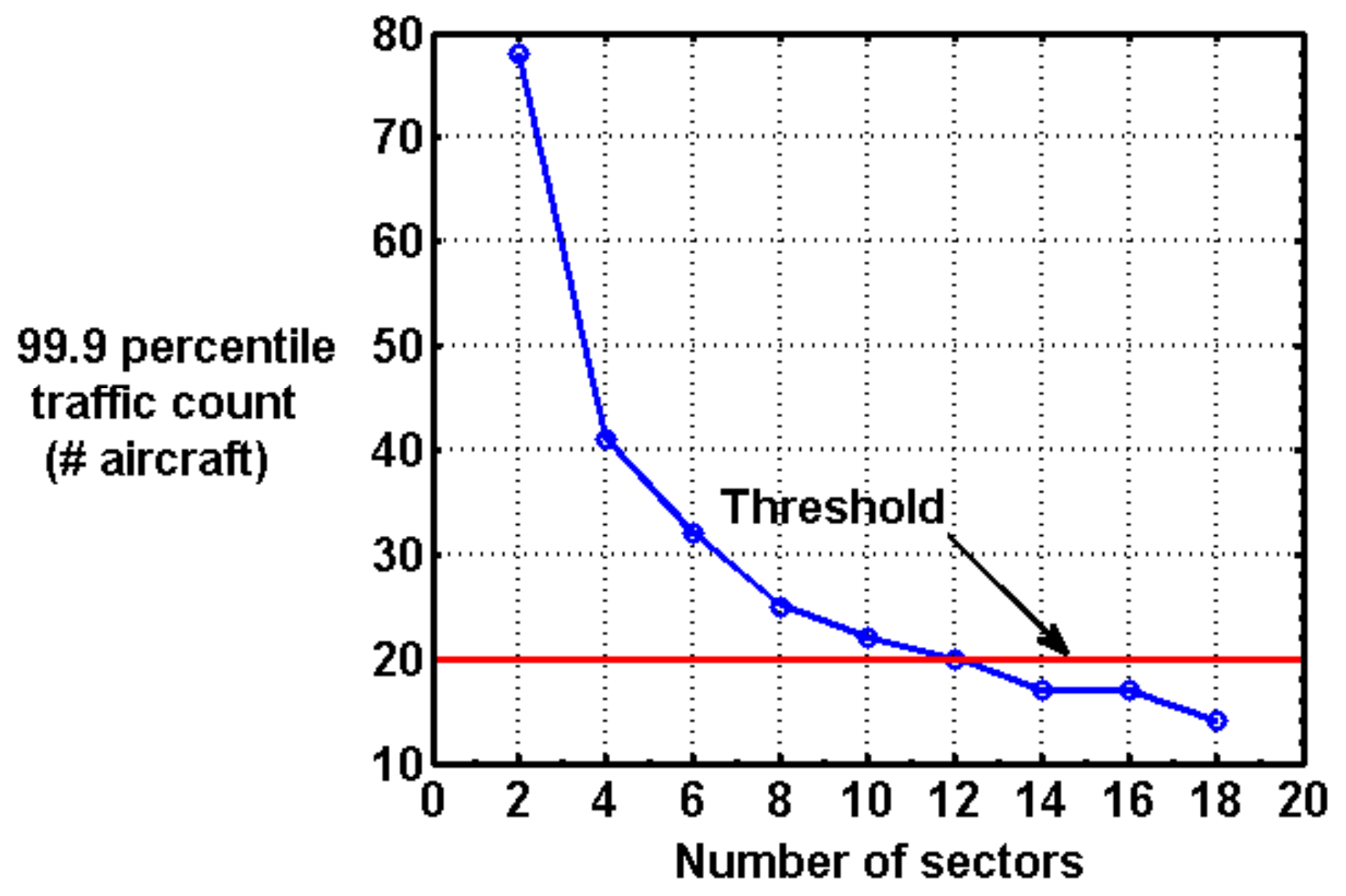

99.9 percentile traffic counts during 6:00 - 8:00 pm Central Standard Time with nine different configurations. 


\section{One Configuration for Each Two-Hour}

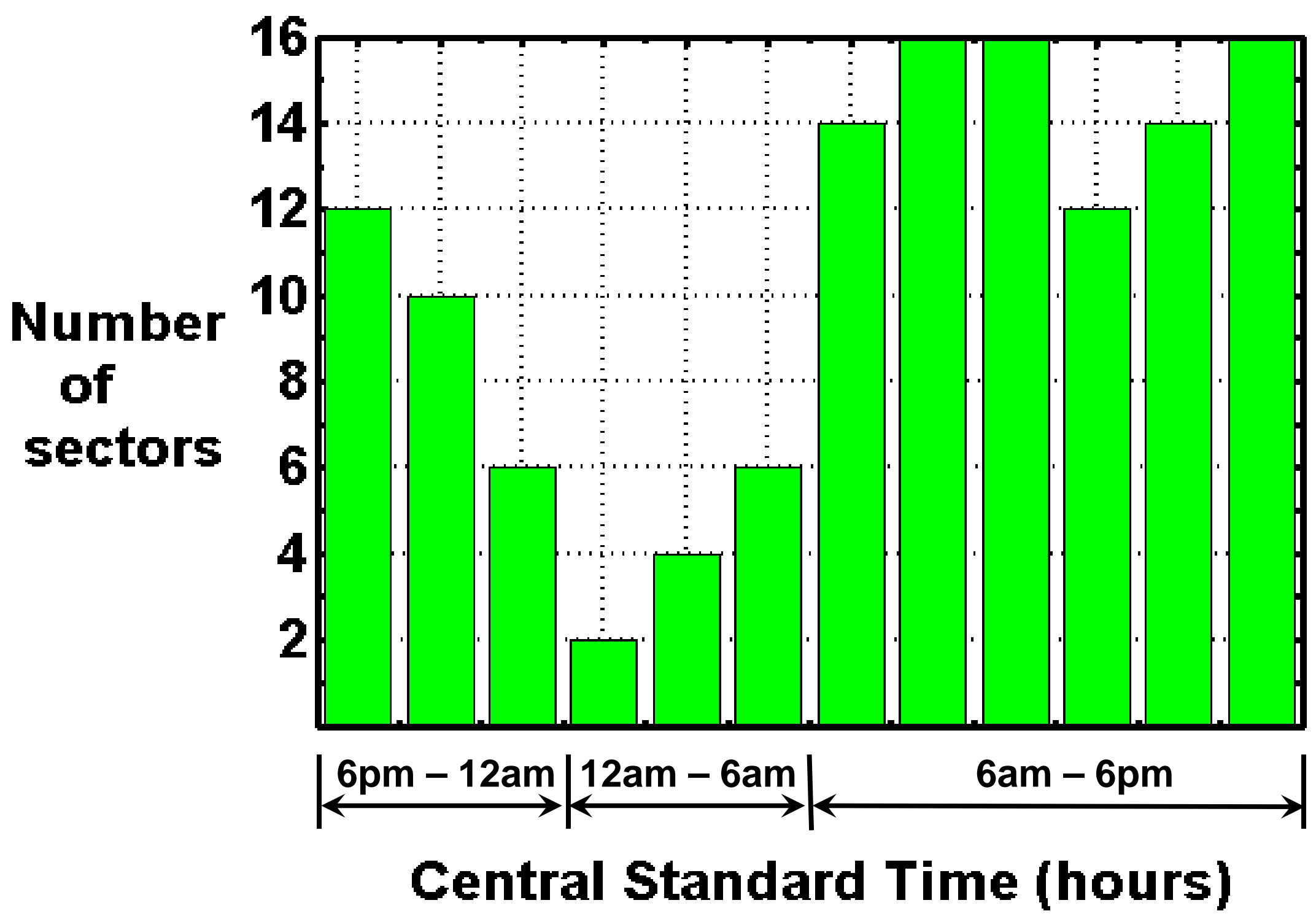




\section{Select Few Configurations}

Three configurations:

6:00 PM - 8:00 PM

4:00 AM - 6:00 AM

10:00 AM - 12:00 Noo

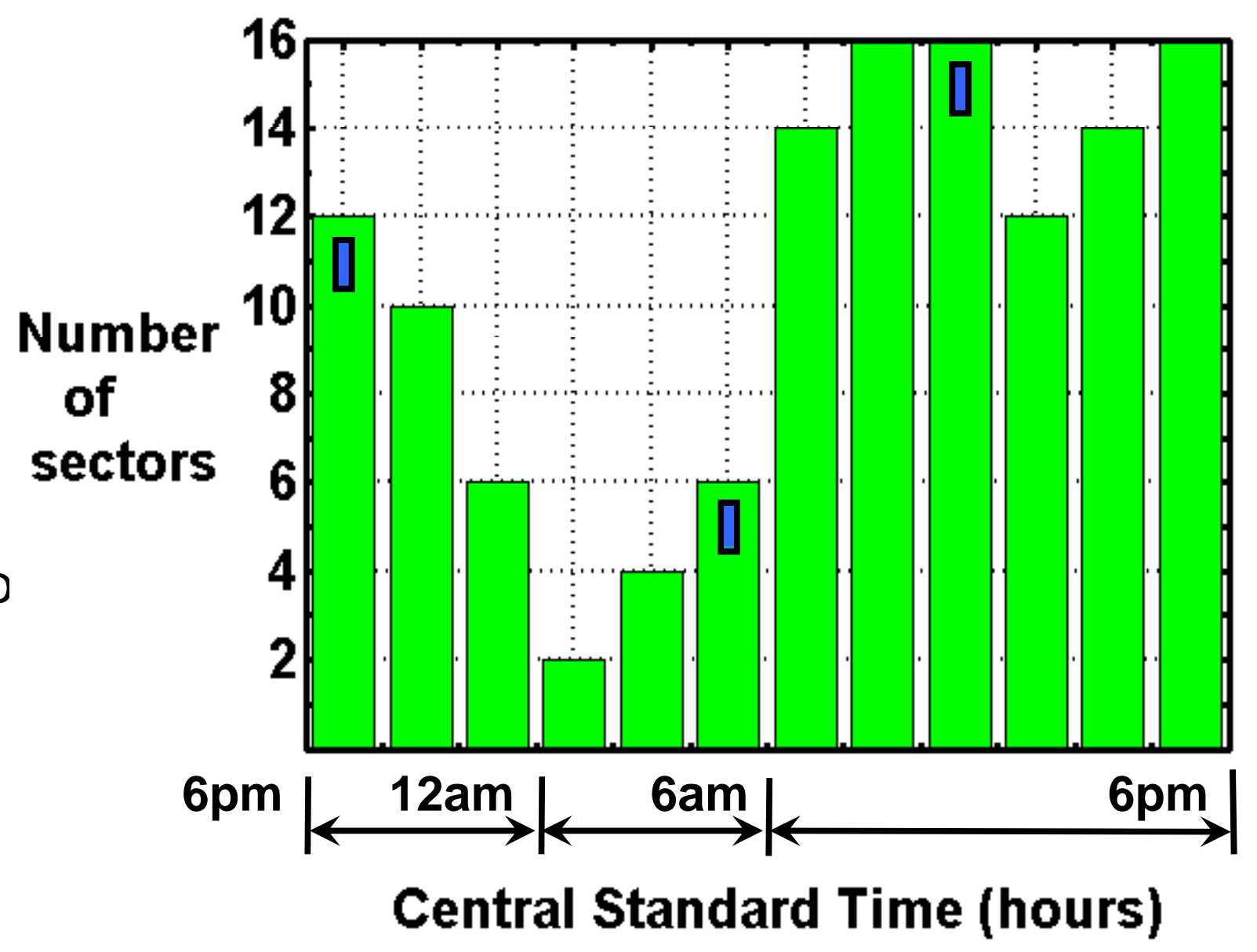




\section{Validation Method}

1. Create histograms using traffic data from test set days and selected sector configurations.

2. Determine 99.9 percentile traffic counts from the histograms.

3. Check if the 99.9 percentile traffic counts are below the threshold value used in the design. 


\section{Validation Results (6:00 PM - 12:00 AM)}

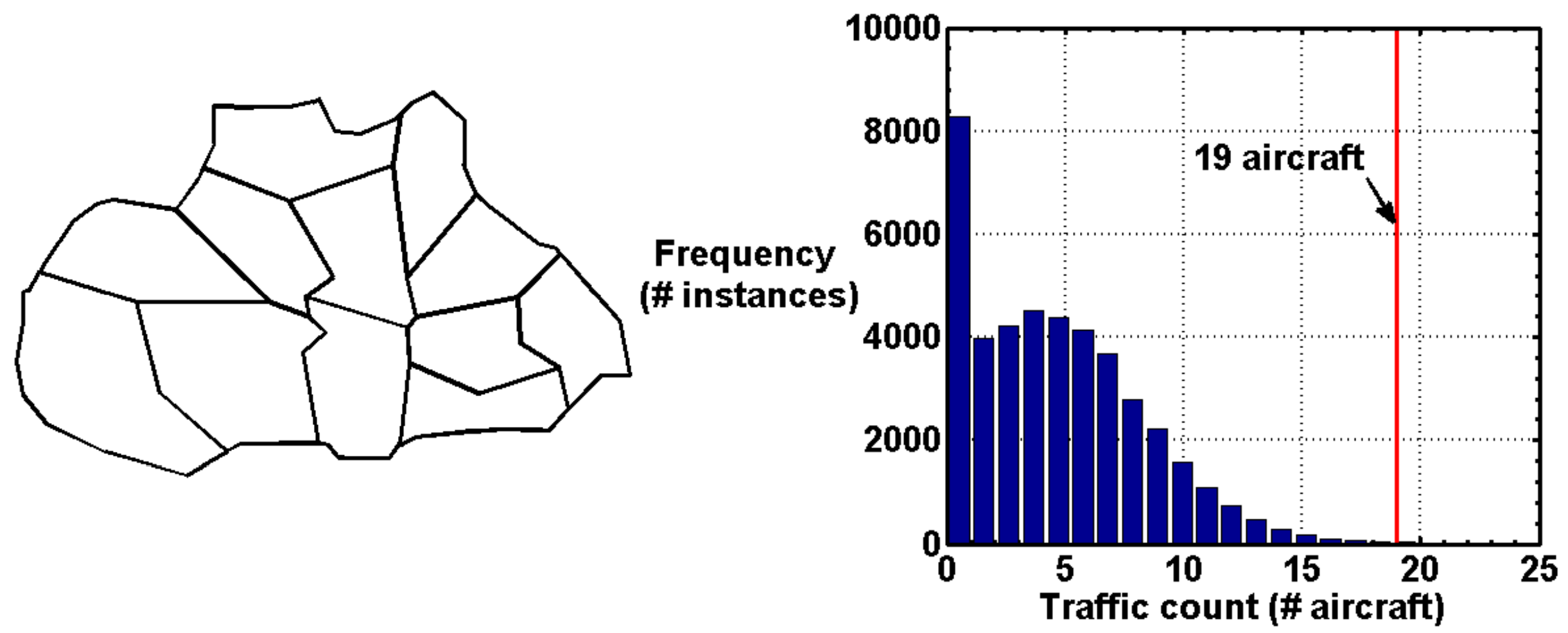

Histogram of traffic counts with 10 test set days and 12 sectors. 


\section{Validation Results (12:00 AM - 6:00 AM)}
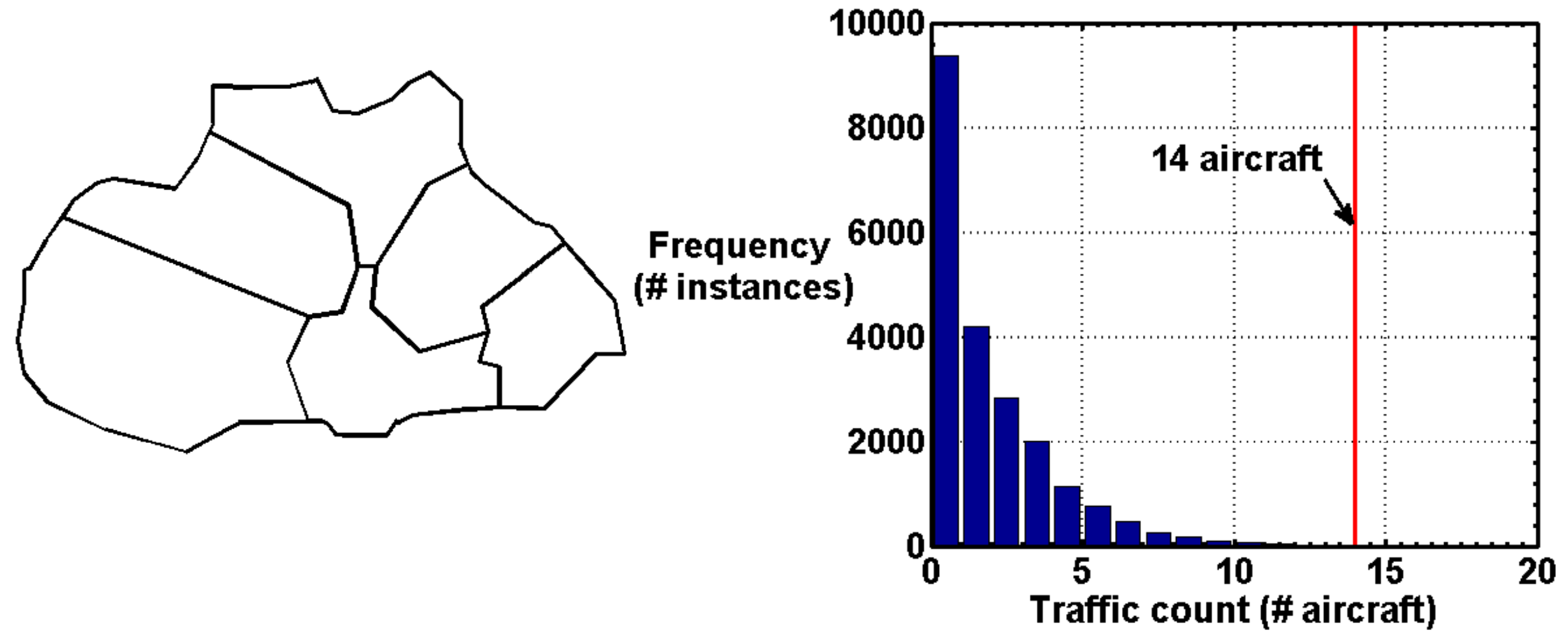

Histogram of traffic counts with 10 test set days and 6 sectors. 


\section{Validation Results (6:00 AM - 6:00 PM)}
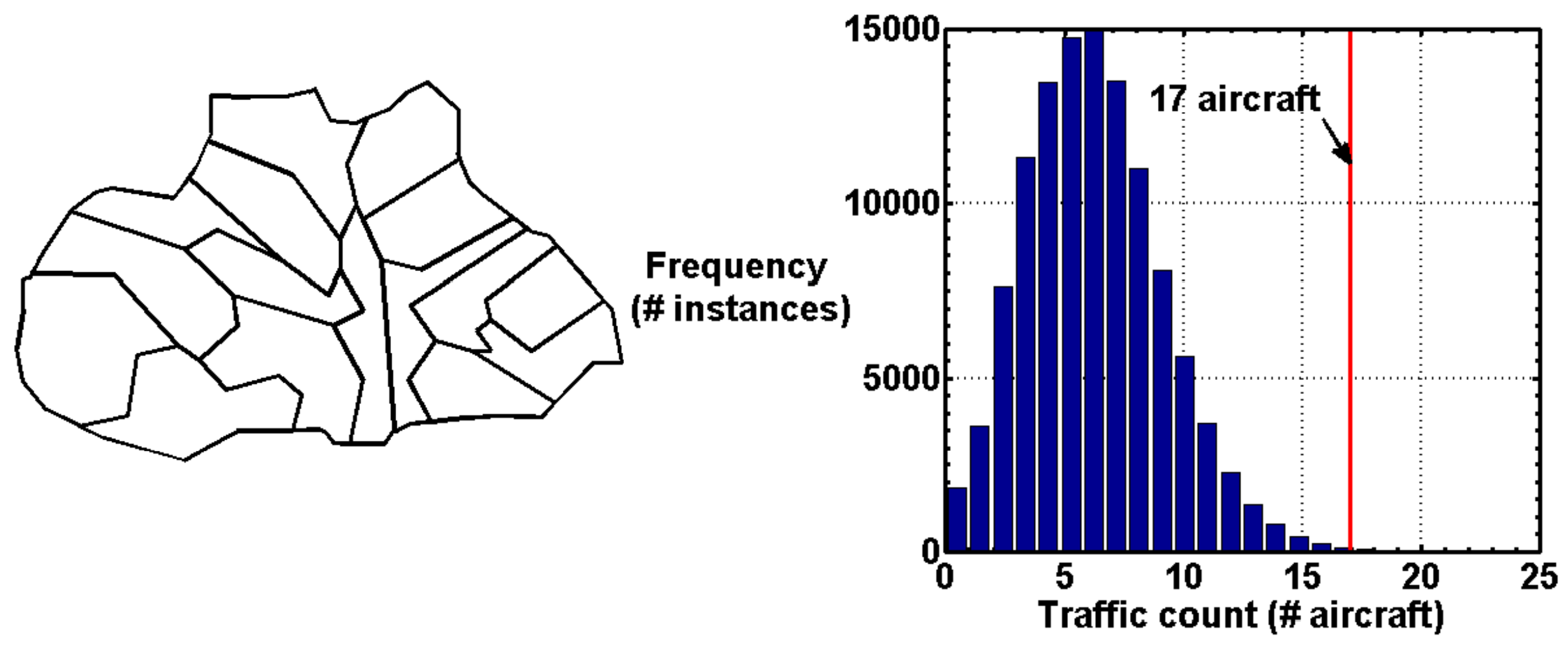

Histogram of traffic counts with 10 test set days and 16 sectors. 


\section{Validation Results (24 hours)}
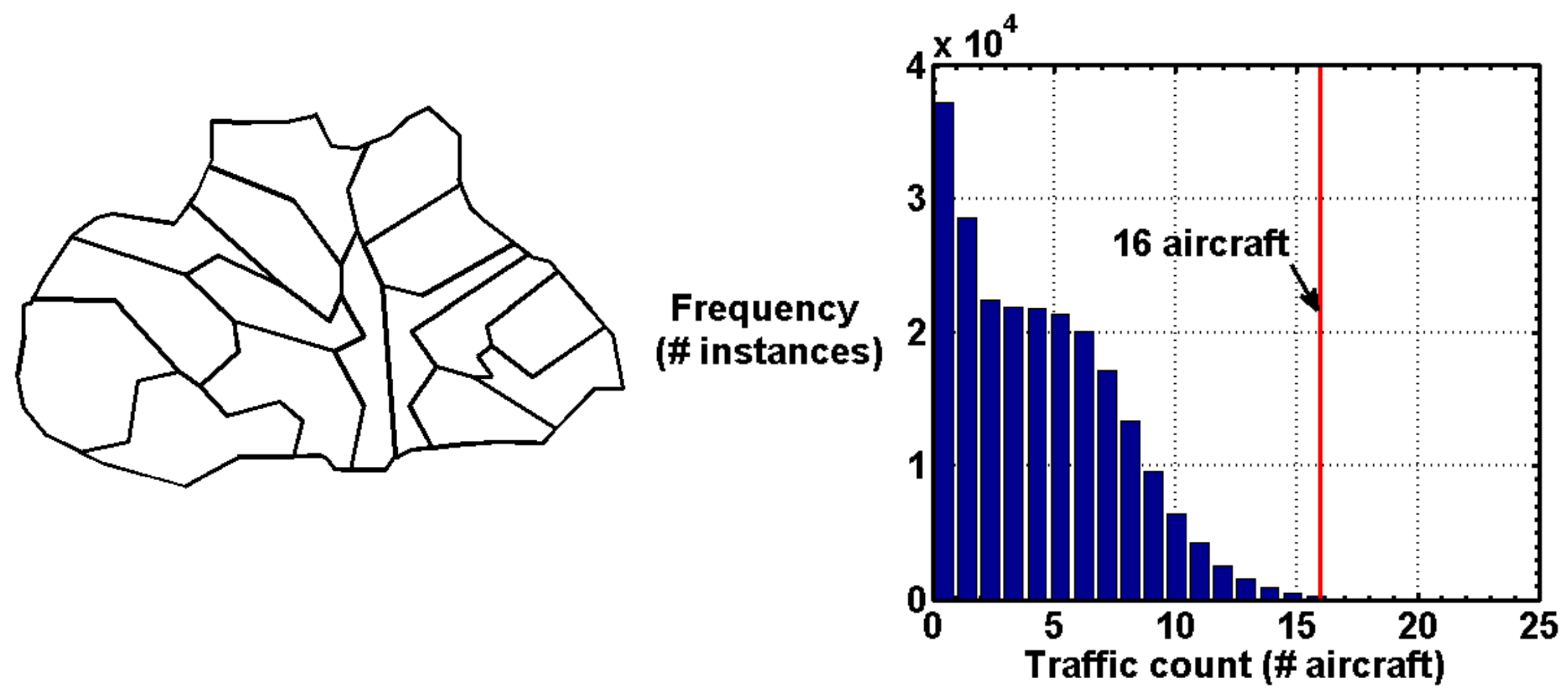

Histogram of traffic counts with 10 test set days and 16 sectors. 


\section{Summary Validation Results}

\begin{tabular}{|l|c|c|c|}
\hline Time Interval & $6 \mathrm{PM}-12 \mathrm{AM}$ & $12 \mathrm{AM}-6 \mathrm{AM}$ & $6 \mathrm{AM}-6 \mathrm{PM}$ \\
\hline $\begin{array}{l}\text { Number of } \\
\text { Sectors }\end{array}$ & 12 & 6 & 16 \\
\hline
\end{tabular}

\begin{tabular}{|l|c|c|c|}
\hline Time Interval & $6 \mathrm{PM}-12 \mathrm{AM}$ & $12 \mathrm{AM}-6 \mathrm{AM}$ & $6 \mathrm{AM}-6 \mathrm{PM}$ \\
\hline Training Days & 19 & 16 & 19 \\
\hline Test Days & 19 & 14 & 17 \\
\hline Test Days & \multicolumn{3}{|c}{16} \\
\hline
\end{tabular}

Design threshold 20 aircraft. 


\section{Conclusions}

- Sectors created with 10 training days of traffic could be used with 10 test days of traffic.

- Sector configurations created for shorter periods (two-hours) could be used for longer periods (six to 12 hours).

- One to three sector configurations were adequate for a day. 


\section{Future Work}

- Extend the study to other centers.

- Analyze with traffic from high-volume bad-weather days.

- Determine the best time for changing configuration. 